\title{
XuefuZhuyu Tang exerts antitumor effects by inhibiting glioma cell metastasis and invasion via regulating tumor microenvironment
}

This article was published in the following Dove Press journal:

OncoTargets and Therapy

17 June 2016

Number of times this article has been viewed

\author{
Jianmin Liu' \\ Ji Zhang ${ }^{2}$ \\ Liangwen Huang' \\ Xuhong Zhu' \\ Wei Chen' \\ Peng $\mathrm{Hu}^{\prime}$ \\ 'Department of Neurosurgery, \\ The First Affiliated Hospital of \\ Guangzhou University of Traditional \\ Chinese Medicine, ${ }^{2}$ Department of \\ Neurosurgery, Sun Yat-sen University \\ Cancer Center, Guangzhou, People's \\ Republic of China
}

Correspondence: Jianmin Liu Department of Neurosurgery, The First Affiliated Hospital of Guangzhou University of Traditional Chinese Medicine, 16 Jichang Road, Baiyun District, Guangzhou 510405,

People's Republic of China

Tel +86 203659 I396

Fax +86 $203659 \quad 1595$

Email jml_liu@।63.com
Background: XuefuZhuyu Tang $(X Z T)$ is a traditional Chinese herb used for destagnation and is currently being used for oncotherapy. This study was intended to assess the effects of $X Z T$ on glioma along with its anticancer mechanism.

Materials and methods: U251 cells were divided into five groups: CNC (cells were cultured with normal saline), TSC (cells were treated with TaohongSiwu Tang [TST]), XSC (cells were treated with $X Z T$ ), THC (cells were treated with homogenate of TST), and XHC (cells were treated with homogenate of $X Z T$ ). The mRNA and protein expression of VEGF/VEGFR, CXCR4/ CXCL12, and TIMP1/MMP9/MMP2 were measured by reverse transcription-polymerase chain reaction (RT-PCR) and Western blotting, respectively. Moreover, MTT assay, transwell assay, wound-healing assay, and flow cytometry were conducted to assess the cell viability, cell migration and invasion, cell motility, and cell apoptosis of U251 cells, respectively. In vivo, three mice models (group CNM, gavaging saline; group TSM, gavaging TST; group XZM, gavaging $X Z T$ ) were constructed after establishing xenograft mice models. Then, models were examined using hematoxylin and eosin staining, RT-PCR, and Western blotting.

Results: In vitro, XZT significantly upregulated TIMP1 expression and downregulated the expression of VEGF, VEGFR, CXCR4, CXCL12, MMP9, and MMP2 in U251 cells (all $P<0.05)$. In addition, $X Z T$ inhibited cell proliferation, invasion, and migration and induced cell apoptosis. In vivo, the average expression level of VEGF, CXCL12, MMP9, and MMP2 was downregulated in the XZM group compared with the control and TSM groups (all $P<0.05$ ). Tumor volumes in the XZM group were significantly lower than those in the CNM and TSM groups (all $P<0.05$ ).

Conclusion: XZT may suppress glioma growth and decrease expression levels of VEGF, CXCL12, MMP9, and MMP2. We speculate that XZT may be a potential therapeutic herb for curing glioma.

Keywords: XuefuZhuyu Tang, glioma, tumor microenvironment, VEGF, CXCL12, TIMP1, MMP9, MMP2

\section{Introduction}

Glioma is commonly discovered in the central nervous system and primarily occurs in the brain and glial tissues. Glioma accounts for $\sim 81 \%$ of malignant brain tumors with unexpectedly high mortality, ${ }^{1}$ and its incidence, though very rare, ranges from 4.67 to 5.73 per 100,000 persons. ${ }^{1}$ It is reported that certain environmental carcinogens, occupations, and diet ( $N$-nitroso compounds) may increase the risk of glioma. ${ }^{2}$ Current treatments for glioma include surgery and concomitant temozolomide chemotherapy. The presence of blood-brain barrier, which prevents macromolecules from entering 
into the brain, limits the efficacy of immunotherapeutic approaches and gene therapy. ${ }^{3}$ Although it has been suspected that tumor growth and progression is driven by the tumor itself, recent studies have suggested that tumor microenvironment (TME) is also associated with tumor progression and metastasis. ${ }^{3}$ TME is a rich and robust stromal cell matrix, which is composed of fibroblasts, endothelial cells, pericytes, leukocytes, and extracellular matrix. Moreover, TEM plays a decisive role in tumor growth and may lead us to develop groundbreaking therapeutic targets for tumors. ${ }^{4}$

Growing studies have attempted to unfold the underlying mechanism between TME and tumor since TME has been acknowledged as a causal factor for the development of tumors. As receptors of CXCL12, both CXCR4 and CXCR7 have been discovered to be highly expressed in tumor cells, which indirectly proved the interplay among stem-like cancer cells, differentiated glioma cells, and TME. Particularly, the circulation is completed via secreted chemokines (eg, CXCL12), which recruit various kinds of molecules, including fibroblasts, endothelial, mesenchymal, and inflammatory cells through CXCR4 to tumor tissues. ${ }^{5}$ Apart from CXCL12, VEGF is believed to be a key growth factor that is responsible for neovasculature; therefore, aberrantly elevated VEGF along with florid vascularization has been considered as a common characteristic of glioma. Meanwhile, it has been suggested that VEGF could regulate tumor invasion by recruiting the protein to a heterocomplex, including VEGFR2, which further suppresses tumor migration. ${ }^{6}$ Recently, antiangiogenic therapies targeting VEGF and its receptors have been developed to inhibit glioma growth. However, the clinical outcomes are transient, and recurrence is usually observed. ${ }^{7}$ MMPs are zinc-dependent endoproteases that are able to degrade the extracellular matrix and mediate tumor growth and metastasis. The tissue inhibitors of metalloproteinases (TIMPs) primarily prevent the matrix from degradation through the inhibition of MMPs. ${ }^{8}$

Traditional Chinese medicine (TCM) has been widely used and practiced for thousands of years. TaohongSiwu Tang (TST) and XuefuZhuyu Tang (XZT) are famous traditional Chinese herbal medicines. XZT includes the following eleven kinds of herbs: Sichuan lovage root (chuanxiong), red peony root (chishao), rehmannia root (shengdi), platycodon root (jiegeng), peach seed (taoren), safflower (honghua), hare's ear root (chaihu), Chinese angelica (danggui), twotoothed achyranthes root (niuxi), orange fruit (zhiqiao), and liquorice. The formulation of XZT is well known for Qi circulation promotion and blood stasis removal. ${ }^{9}$ Many herbs in the medicine belong to plant functional foods and play protective roles in cancer prevention/treatment..$^{10,11}$ Since tetramethylpyrazine (TMP) extracted from the Chinese herb chuanxiong has positive effects on tumor care, $X Z T$ is considered as a treatment for cardiovascular and chronic liver diseases. ${ }^{9,12}$ The effects of $X Z T$ on fibrotic liver include not only the inhibition of collagen deposition but also the antiangiogenesis. ${ }^{12}$

Though a large number of studies have revealed the antiangiogenetic effects of $X Z T$, the molecular mechanisms of $X Z T$ that are related to glioma are still ambiguous. As a result of this, we used both in vitro and in vivo models to study the effect of $X Z T$ on tumor cells and expressions of VEGF, CXCL12, MMP9, and MMP2. We suspected that $X Z T$ inhibits glioma development and metastasis by regulating the extracellular microenvironment of glioma cells, which may further provide new insights for glioma therapies.

\section{Materials and methods}

\section{Animals, cells, and groups}

Herbs of TST and XZT used in this study were purchased from The First Affiliated Hospital of Guangzhou University (Guangzhou, People's Republic of China). Then dry herbs were dissolved in sterile $0.9 \% \mathrm{NaCl}$ to form appropriate concentrations for usage. Normal saline was applied as the control in all experiments.

Sixteen male SD rats with an average weight of $\sim 120 \mathrm{~g}$ and 24 male BALB/c nude mice with an average weight of $\sim 10 \mathrm{~g}$ were purchased from the Laboratory Animal Center of Southern Medical University (Guangzhou, People's Republic of China). Animals were housed in a specific pathogen-free environment. The relative humidity and temperature were set at $50 \% \pm 10 \%$ and $25^{\circ} \mathrm{C} \pm 1^{\circ} \mathrm{C}$, respectively. Animals were subjected to 10 hour light and 14 hour dark cycles per 24 hours.

U251 glioma cell lines were offered by the Institute of Biochemistry and Cell Biology (Shanghai, People's Republic of China). Cells were grown at $37^{\circ} \mathrm{C}$ in Roswell Park Memorial Institute 1640 medium with 10\% fetal bovine serum, $100 \mathrm{U} / \mathrm{mL}$ penicillin, and $100 \mathrm{mg} / \mathrm{L}$ streptomycin in a humidified atmosphere containing $5 \% \mathrm{CO}_{2}$.

Cultured cells were divided into the following five groups: group CNC (U251 cells cultured with normal saline, control group), group TSC (U251 cells cultured with serum of TST-treated SD rats, medicine control group), group XSC (U251 cells cultured with serum of XZT-treated SD rats), group THC (U251 cells cultured with homogenate of TSTtreated SD rats), and group XHC (U251 cells cultured with homogenate of $X Z T$-treated SD rats). The above experiments 
were carried out for 1 week. This study and all study protocols were approved by the ethics committee of The First Affiliated Hospital of Guangzhou University of Traditional Chinese Medicine, and this study complied with the Guidelines for Animal Experimentation Committee at Guangzhou University of Traditional Chinese Medicine.

\section{RNA extract and real-time reverse transcription-polymerase chain reaction} The mRNAs of CXCR4, CXCL12, VEGF, VEGFR, TIMP1, MMP9, and MMP2 were taken from cells and tissues using the miRNeasy Mini Kit (Qiagen NV, Venlo, the Netherlands) under the manufacturer's instructions. Reverse transcriptionpolymerase chain reaction (RT-PCR) was performed to obtain cDNA template using the ReverTra Ace qPCR RT Kit (Toyobo, Osaka, Japan) along with the CFX96 Touch RT-PCR Detection System (Bio-Rad Laboratories Inc., Hercules, CA, USA). The primer sequences are shown in Table 1. Relative expression quantity of target genes was normalized to that of the internal control group ( $\beta$-actin) and was calculated using the $2^{-\Delta \Delta \mathrm{CT}}$ method. The experimental procedures were repeated for at least three times.

\section{Western blotting}

Western blotting was used to detect the expression level of targeted proteins. Cellular extracts were lysed by the lysis buffer used in radioimmunoprecipitation assay. Supernatant was collected after centrifuging and lysing the cellular extracts at $12,000 \mathrm{rpm}$ for 15 minutes at $4^{\circ} \mathrm{C}$. Cell proteins were isolated using sodium dodecyl sulfate-polyacrylamide gels and were transferred to polyvinylidenedifluoride membranes (Bio-Rad Laboratories Inc.). Then membranes with isolated cell proteins were blocked for 1 hour and detected using primary antibodies, including anti-CXCR4 (1:100), anti-CXCL12 (1:100), antiVEGF (1:500), anti-VEGFR (1:500), anti-TIMP1 (1:300), anti-MMP9 (1:300), anti-MMP2 (1:300), and anti- $\beta$-actin (1:100) (Santa Cruz Biotechnology Inc., Dallas, TX, USA) at $4^{\circ} \mathrm{C}$ overnight. Membranes were further washed thrice by $\times 1$ phosphate-buffered saline (PBS) Tween-20 (10 min/time). After that, horseradish peroxidase-conjugated secondary antibodies were added to membranes, which were incubated for another 1 hour. Finally, membranes were washed thrice again using Tris buffered saline Tween (10 min/time). The immunobinding signals were detected by the chemiluminescence method (KeyGen Biotech Co. Ltd, Nanjing, People's Republic of China). Relative protein expression was subjected to densitometry analysis using the Image-Pro Plus Version 6.0 software (Media Cybernetics., Inc, Washington, IL, USA) and calculated according to the $\beta$-actin reference bands.

\section{MTT assay}

MTT (3-(4,5-dimethyl-2-thiazolyl)-2,5-diphenyl-2-Htetrazolium bromide) assay was carried out to assess the viability of U251 cells. U251 glioma cells were incubated for 4 hours at $37^{\circ} \mathrm{C}$ in a 96 -well plate, and then $0.5 \%$ DMSO was added to each well in the plate. Absorbance was detected by a fluorescence plate reader at $560 \mathrm{~nm}$ (Bio-Rad Laboratories Inc.). Cell viability was determined by the optical density ratio of a treated group over an untreated control group. The experiments were repeated for three times.

Table I Primer sequences of targeted genes

\begin{tabular}{|c|c|c|}
\hline Gene & Primer & Size (bp) \\
\hline \multirow[t]{2}{*}{ CXCR4 } & For 5'-ACTCGGTGCTCGGAGTATG-3' & 558 \\
\hline & Rev 5'-TGGTCAGTCTTGTTTTTCTGAGAT-3' & \\
\hline \multirow[t]{2}{*}{ CXCLI2 } & For 5'-GTCGCCAGAATGGACCTC-3' & 1,180 \\
\hline & Rev 5'-CTTGGGAGAACGGCTCTTTA-3' & \\
\hline \multirow[t]{2}{*}{ VEGF } & For 5'-TTGCTGCTCTACCTCCACCAT-3' & 64 \\
\hline & Rev 5'-TGTGCTCTССTССTGCCATAG-3' & \\
\hline \multirow[t]{2}{*}{ VEGFR } & For 5'-TGAAGGAAGGCAGATCGTCAT-3' & 111 \\
\hline & Rev 5'-CAGGTTATTCGCTTCCCATCA-3' & \\
\hline \multirow[t]{2}{*}{ TIMPI } & For 5'-TGCACCTGTGTCCCACCCCACCCACAGACG-3' & 551 \\
\hline & Rev 5'-GGCTATCTGGGACCGCAGGGACTGCCAGGT-3' & \\
\hline \multirow[t]{2}{*}{ MMP9 } & For 5'-GTTCCCGGAGTGAGTTGA-3' & 109 \\
\hline & Rev 5'-TTTACATGGCACTGCAAAGC-3' & \\
\hline \multirow[t]{2}{*}{ MMP2 } & For 5'-CCACTGCCTTCGATACAC-3' & 133 \\
\hline & Rev 5'-GAGCCACTCTCTGGAATCTTAAA-3' & \\
\hline \multirow[t]{2}{*}{$\beta$-Actin } & For 5'-TCGACAACGGCTCCGGCAT-3' & $24 I$ \\
\hline & Rev 5'-AAGGTGTGGTGCCAGATTTTC-3' & \\
\hline
\end{tabular}

Abbreviations: For, forward; Rev, reverse. 


\section{Transwell assay}

A transwell chamber $(8 \mathrm{~mm}$ pore size; Costar, High Wycombe, UK) was placed into the wells of 12-well culture plates in order to measure cell migration and invasion. A total of $5 \times 10^{4}$ cells were added to the upper chamber and cultured with serum-free medium, while the lower chamber was filled with the complete medium. Cells in the upper chamber were carefully removed by a cotton swab after 24 -hour incubation, while cells migrated or invaded into the lower surface were fixed and stained with eosin. Matrigel was specifically added to the medium for cell invasion assay. The number of migrated or invaded cells was counted for five random fields under the microscope (Olympus Corporation, Tokyo, Japan), and three independent assays were conducted.

\section{Wound-healing assay}

The wound-healing assay was conducted to assess cell motility. U251 cells in each group were seeded into 12-well plates 1 day prior to scratching. A scratching wound was performed by scraping the middle of the cell monolayer using a sterile micropipette tip. After removing the detached cells by PBS, cells were cultured with complete medium. Image of cells migrating into the wound was captured at 0 hour and 24 hours using a microscope recording the distance of U251 cell migration.

\section{Cell apoptosis analysis}

Apoptosis was evaluated using the Annexin V-FITC Apoptosis Detection Kit. U251 cells were seeded in six-well plates, incubated overnight, and then treated with serum (TST or $X Z T$ ) and homogenate (within TST or XZT) for 3 days. Cells were harvested by trypsinization, washed with PBS, and finally centrifuged. Cell pellet was resuspended in the binding buffer and incubated with Annexin V-fluorescein isothiocyanate (FITC). After that, cells were again centrifuged, washed with PBS, and resuspended in the binding buffer containing PI solution in the dark. Finally, cells were analyzed by the approach of flow cytometry (Beckman FC400 MPL; Beckman Coulter Inc., California, IL, USA). Each experiment was independently carried out in triplicate.

\section{Xenograft mouse model}

U251 cells were harvested, washed with PBS, and resuspended in a mixture of culture medium and Matrigel (BD Biosciences, San Jose, CA, USA). Then, U251 cells ( $1 \times 10^{6}$ per site) were subcutaneously implanted into the two flanks of all nude mice.

A total of 24 mice were randomly allocated into the following three groups with equal size when tumors grew to the level of $200 \mathrm{mg}$ : group CNM (gavaging saline), group TSM (gavaging TST), and group XZM (gavaging XZT). Treatments were administered once a day for the period of 28 days, and the concentrations of TST and XZT were both set at $100 \mu \mathrm{M}$.

Tumor size in the two flanks was measured twice per week using calipers (tumor volume $=\left[\right.$ length $\times$ width $\left.^{2}\right] \times 0.5$ ). Tumor growth was monitored twice per week for the period of 30 days. Mice were anesthetized and sacrificed at the end of the experiment, and tumors were extracted from mice for imaging and weighing. Tumors were fixed with $4 \%$ paraformaldehyde, dehydrated, and embedded with paraffin. Then, $5 \mathrm{~mm}$ sections were cut from tumor tissues and stained with hematoxylin and eosin and observed using a microscope. The experimental procedures were complied with the Animal Management Rule of the Chinese Ministry of Health (Documentation 55, 2001), and the experimental protocol was approved by the ethics committee of The First Affiliated Hospital of Guangzhou University of Traditional Chinese Medicine.

\section{Statistical analysis}

All statistical analyses were performed by SPSS 18.0 software (SPSS Inc., Chicago, IL, USA). Continuous data were expressed in the form of mean $\pm \mathrm{SD}$. The two-tailed Student's $t$-test or one-way analysis of variance was used to assess between-group comparisons, and $P<0.05$ provided evidence for statistical significance.

\section{Results $X Z T$ affects expressions of VEGF/VEGFR, CXCR4/CXCLI2,TIMPI/MMP9/MMP2 in U25 I glioma cells in vitro}

Both RT-PCR and Western blotting were conducted to assess whether $X Z T$ is able to affect the expression of VEGF/VEGFR, CXCR4/CXCL12, and TIMP1/MMP9/MMP2 in glioma U251 cells. As shown in Figure 1, the TIMP1 mRNA expression level in U251 cells treated with $X Z T$ was significantly higher than that in U251 cells treated with saline or TST, while the mRNA expression levels of VEGF, VEGFR, CXCR4, CXCL12, MMP9, and MMP2 exhibited the opposite trend $(P<0.05)$. Similarly, Western blotting results indicated that TIMP1 in U251 cells treated with XZT expressed significantly higher than that in U251 cells treated with saline or TST, while the expressions of VEGF, VEGFR, CXCR4, CXCL12, MMP9, and MMP2 in U251 cells treated with XZT were significantly lower than those in U251 cells treated with saline or TST $(P<0.05)$ (Figure 2). Our data revealed that $X Z T$ significantly upregulated the expression of TIMP1 and 
A
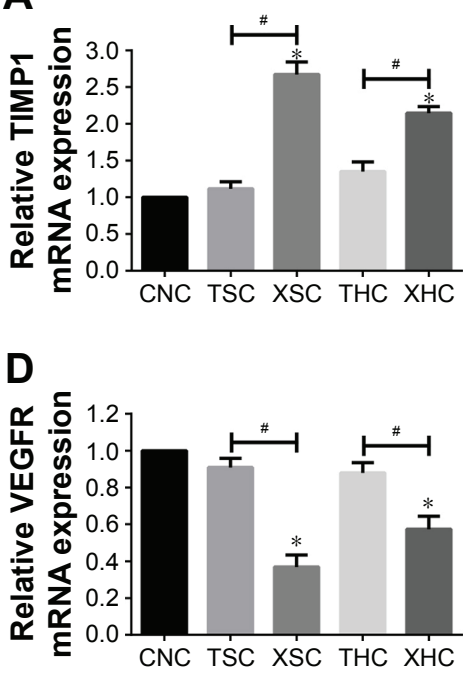

B

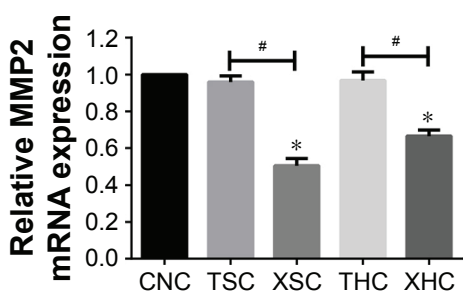

E

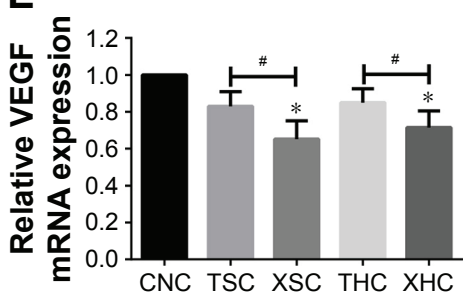

C

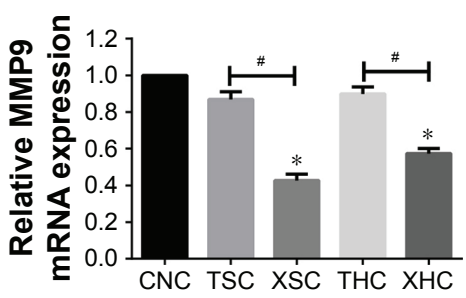

$\mathbf{F}$

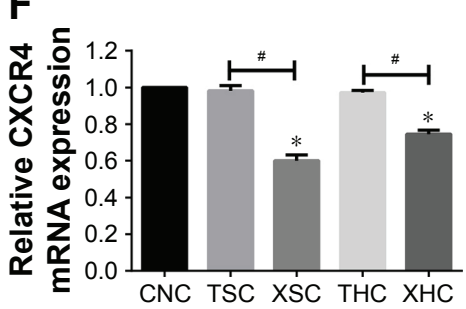

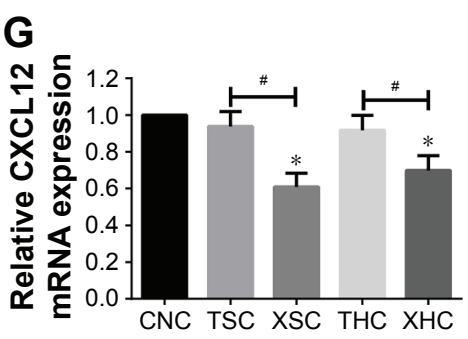

Figure I The relative mRNA expression of targeted genes was measured by RT-PCR in U25 I cells with different treatments.

Notes: (A) TIMPI, (B) MMP2, (C) MMP9, (D) VEGFR, (E) VEGF, (F) CXCR4, and (G) CXCLI2. *Versus group CNC, $P<0.05$; ${ }^{*}$ Group XSC versus group TSC and group $X H C$ versus group THC, $P<0.05$. Group CNC: U25I cells were cultured with normal saline, control group. Group TSC: U25I cells were cultured with serum of SD rats treated with TST, medicine control group. Group XSC: U25I cells were cultured with serum of SD rats treated with XZT. Group THC: U25I cells were cultured with serum of SD rats treated with homogenate of TST. Group XHC: U25I cells were cultured with serum of SD rats treated with homogenate of XZT.

Abbreviations: RT-PCR, reverse transcription-polymerase chain reaction; SD, Sprague Dawley; TST, TaohongSiwu Tang; XZT, XuefuZhuyu Tang.

downregulated the expression of VEGF, VEGFR, CXCR4, CXCL12, MMP9, and MMP2 in U251 glioma cells in vitro.

\section{$X Z T$ inhibits tumor cell proliferation of U25 I glioma cell lines in vitro}

Furthermore, the MTT assay was performed to evaluate the viability of $\mathrm{U} 251$ glioma cells treated with XZT, TST, and saline. Briefly, cells were treated with five different treatment groups for a period of 24 hours, 48 hours, and 72 hours. As suggested by Figure 3, XZT inhibited U251 glioma cell proliferation in a time-dependent manner. Moreover, the inhibition rate in the XSC group was significantly higher than that in the $\mathrm{CNC}$ and TSC groups when cells were treated for a period of 48 hours or 72 hours (all $P<0.05$ ).

\section{$X Z T$ inhibits glioma cell invasion and migration}

As suggested by Figure 4, the number of migrated cells in group XSC (80.12 \pm 4.13$)$ was significantly less than that in group CNC (189.58 \pm 10.05$)$ or group TSC (149.685 \pm 5.16$)$ (all $P<0.05$ ) after treatments have been applied for a period of 7 days, suggesting that $X Z T$ serum can effectively inhibit U251 glioma cell migration. Besides that, $X Z T$ homogenate served as a glioma cell migration inhibitor. The number of migrated cells in group XHC (79.78 \pm 6.20$)$ was significantly less than those in group CNC $(189.58 \pm 10.05)$ and group THC (166.44 \pm 15.02$)$ (all $P<0.05$, Figure 4). As shown in Figure 5, results from wound-healing assays illustrated that group XSC suppressed cell migration after treatments have been applied for 12 hours, and the same trend was observed after treatments have been applied for 24 hours.

\section{$X Z T$ induces apoptosis of U25 I glioma cells in vitro}

Flow cytometry with Annexin V-FITC/PI staining was used to analyze whether $X Z T$ was able to induce $\mathrm{U} 251$ glioma cell apoptosis. As suggested by Figure $6 \mathrm{~A}-\mathrm{C}$, the apoptosis rate in group XSC was significantly higher compared to that in group CNC or TSC (all $P<0.05$ ), which indicated that $X Z T$ serum can greatly promote $\mathrm{U} 251$ glioma cell apoptosis. $X Z T$ 


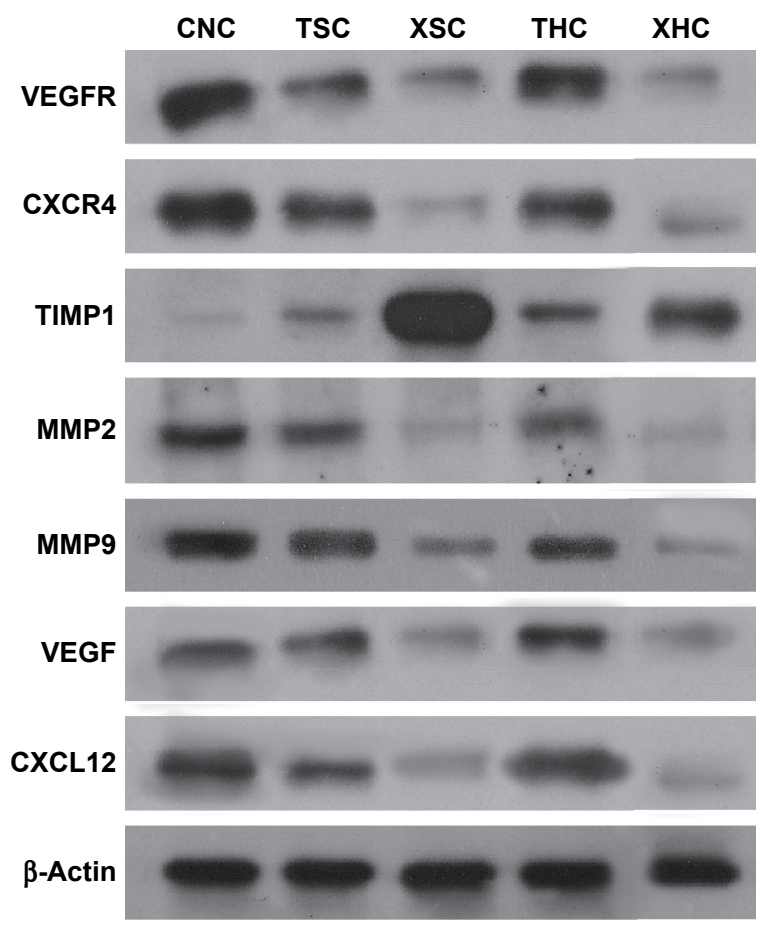

Figure 2 The expressions of targeted proteins in U25I cells with different treatments by Western blotting.

Notes: $\beta$-Actin was used as control. Group CNC: U25I cells were cultured with normal saline, control group. Group TSC: U25I cells were cultured with serum of SD rats treated with TST, medicine control group. Group XSC: U25I cells were cultured with serum of SD rats treated with XZT. Group THC: U25I cells were cultured with serum of SD rats treated with homogenate of TST. Group XHC: U25I cells were cultured with serum of SD rats treated with homogenate of $X Z T$. Abbreviations: SD, Sprague Dawley; TST, TaohongSiwu Tang; XZT, XuefuZhuyu Tang.

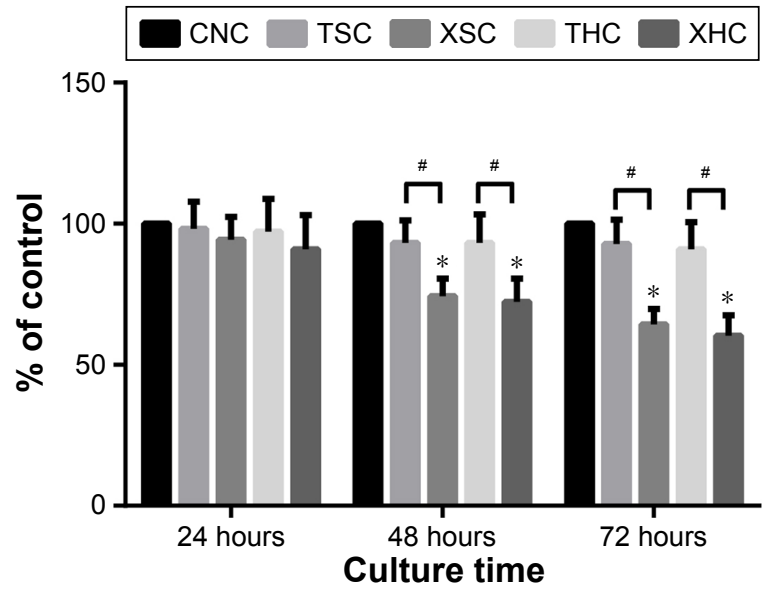

Figure $3 X Z T$ inhibits U25I glioma cell proliferation detected by the MTT assay. Notes: The five groups of cells were cultured for 24 hours, 48 hours, and 72 hours. Results are expressed relative to the cell density of group CNC. *Versus group $\mathrm{CNC}, \mathrm{P}<0.05$; " Group $\mathrm{XSC}$ versus group TSC and group $\mathrm{XHC}$ versus group THC, $P<0.05$. Group CNC: U25I cells were cultured with normal saline, control group. Group TSC: U25I cells were cultured with serum of SD rats treated with TST, medicine control group. Group XSC: U25I cells were cultured with serum of SD rats treated with XZT. Group THC: U25I cells were cultured with serum of SD rats treated with homogenate of TST. Group XHC: U25I cells were cultured with serum of SD rats treated with homogenate of $X Z T$.

Abbreviations: MTT,3-(4,5-dimethyl-2-thiazolyl)-2,5-diphenyl-2-H-tetrazolium bromide; SD, Sprague Dawley; TST, TaohongSiwu Tang; XZT, XuefuZhuyu Tang.

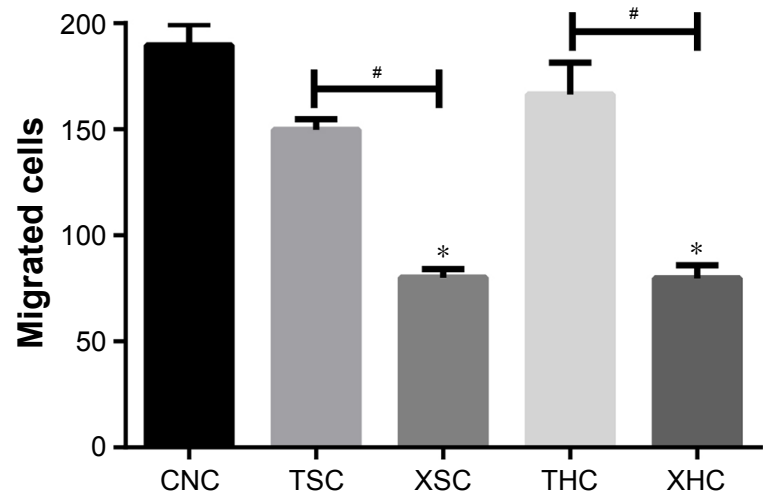

Figure 4 The invasion and migration ability of U25I glioma cells at 7 days after treatment was detected by transwell assay.

Notes: *Versus group CNC, $P<0.05$; ${ }^{*}$ Group $X S C$ versus group TSC and group $\mathrm{XHC}$ versus group THC, $P<0.05$. Group CNC: U25I cells were cultured with normal saline, control group. Group TSC: U25I cells were cultured with serum of SD rats treated with TST, medicine control group. Group XSC: U25I cells were cultured with serum of SD rats treated with XZT. Group THC: U25I cells were cultured with serum of SD rats treated with homogenate of TST. Group XHC: U25I cells were cultured with serum of SD rats treated with homogenate of XZT.

Abbreviations: SD, Sprague Dawley; TST, TaohongSiwu Tang; XZT, XuefuZhuyu Tang.

homogenate also showed a similar tendency: the apoptosis rate in group $\mathrm{XHC}$ was significantly higher than those in group CNC or THC (all $P<0.05$, Figure $6 \mathrm{~A}, \mathrm{D}$, and E).

\section{XZT suppresses glioma cell proliferation in vivo}

Volumes of each xenograft tumor were measured after they were extracted from the xenograft mice. As displayed

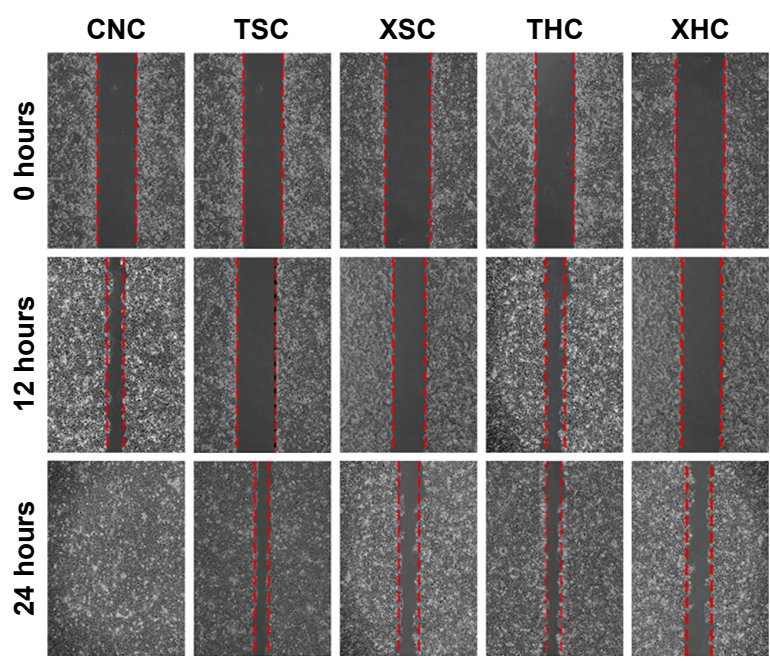

Figure 5 The scratch repaired ability of $U 25$ I glioma cells at I 2 hours and 24 hours after treatment was detected by wound-healing assay.

Notes: Group XSC was compared with group CNC and group TSC, and group $\mathrm{XHC}$ was compared with group CNC and group THC. Group CNC: U25I cells were cultured with normal saline, control group. Group TSC: U25I cells were cultured with serum of SD rats treated with TST, medicine control group. Group XSC: U25I cells were cultured with serum of SD rats treated with XZT. Group THC: U25I cells were cultured with serum of SD rats treated with homogenate of TST. Group XHC: U25I cells were cultured with serum of SD rats treated with homogenate of $X Z T$.

Abbreviations: SD, Sprague Dawley; TST, TaohongSiwu Tang; XZT, XuefuZhuyu Tang. 

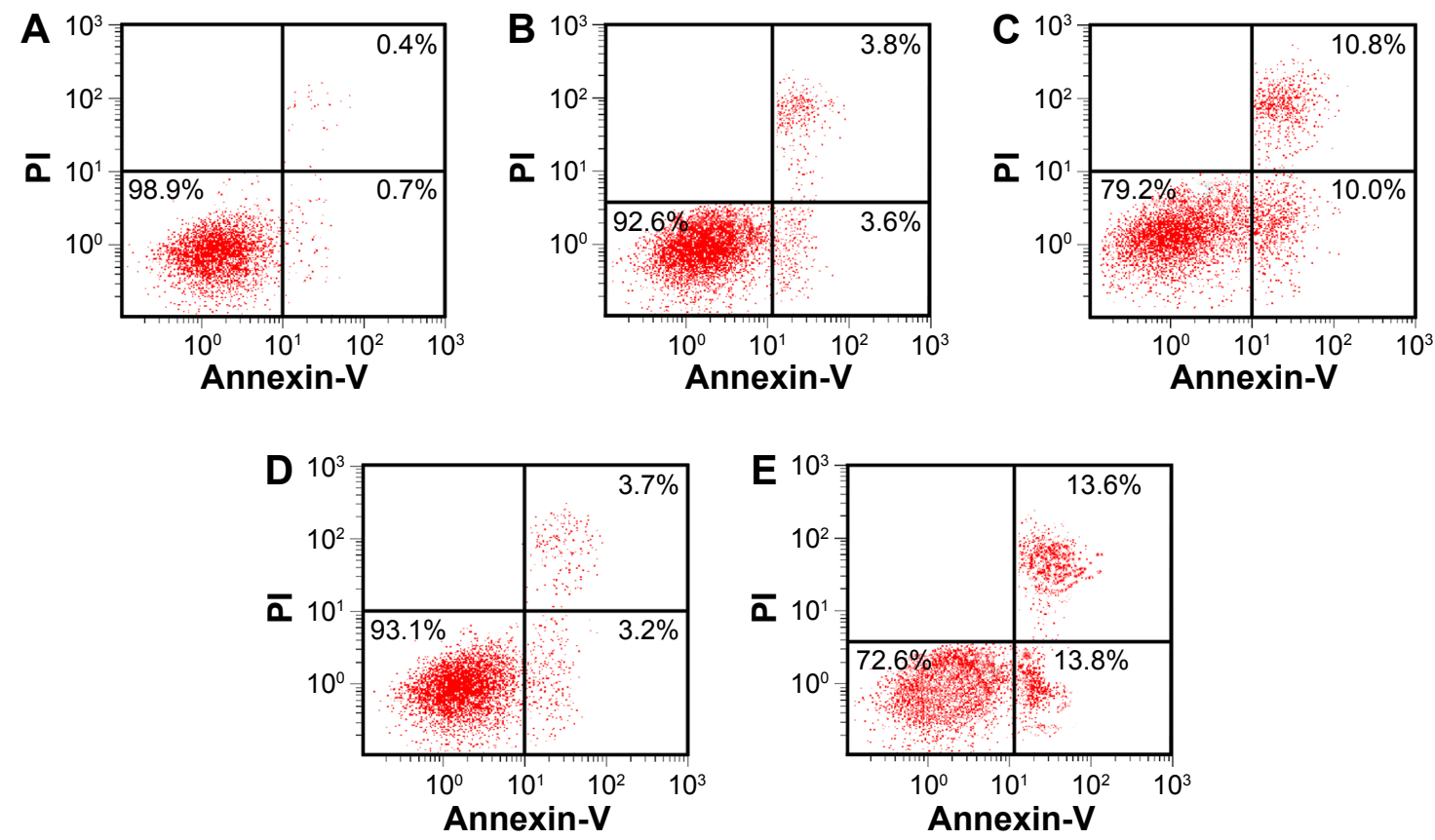

Figure 6 The apoptosis of U25I cells in different groups tested by flow cytometry through Annexin V/PI double staining.

Notes: (A) Group CNC, (B) group TSC, (C) group XSC, (D) group THC, and (E) group XHC. Group CNC: U25I cells were cultured with normal saline, control group. Group TSC: U25I cells were cultured with serum of SD rats treated with TST, medicine control group. Group XSC: U25I cells were cultured with serum of SD rats treated with XZT. Group THC: U25I cells were cultured with serum of SD rats treated with homogenate of TST. Group XHC: U25I cells were cultured with serum of SD rats treated with homogenate of $X Z T$.

Abbreviations: PI, propidium iodide, SD, Sprague Dawley; TST, TaohongSiwu Tang; XZT, XuefuZhuyu Tang.

in Figure $7 \mathrm{~A}$ and $\mathrm{B}$, tumor volumes in group XZM $\left(542.25 \pm 84.51 \mathrm{~mm}^{3}\right)$ were significantly lower than those in group CNM $\left(1,230.33 \pm 164 \mathrm{~mm}^{3}\right)$ and group TSM $\left(1,052.63 \pm 156.89 \mathrm{~mm}^{3}\right)($ all $P<0.05)$ after treatments had been applied for a period of 30 days. Moreover, we conducted hematoxylin and eosin stain to observe the morphology of tumors (Figure 7C). There was no significant abnormality observed in tumor cells of group CNM. However, tumor cells in group TSM and group XZM migrated into the brain to some extent, particularly glioma cells in group XZM showed a higher migration pattern (Figure $7 \mathrm{C}$ ), indicating that glioma cells treated with $X Z T$ for a period of 30 days may be associated with a stronger suppression of cell proliferation compared to that in group CNM or group TSM. As suggested by Figure 7D, RT-PCR and Western blotting in vitro revealed that expressions of VEGF, CXCL12, MMP9, and MMP2 were downregulated in XZT group in comparison to the control and TST groups.

\section{Discussion}

As a common brain neoplasm, glioma has poor prognosis due to the lack of effective treatments. ${ }^{13}$ So far, the median survival time of patients with malignant glioma is $\sim 1$ year. ${ }^{13}$ A variety of studies both in vivo and in vitro have demonstrated that TCM, such as Chinese herbs, may be considered as alternative treatments for cancer. ${ }^{14,15}$ For instance, TMP extracted from chuanxiong, which is a natural Chinese herb, has been suggested as a potential therapy for glioma due to its neural protection actions. ${ }^{16-18}$

$X Z T$ is a widely known TCM formulation consisting of eleven herbal substances, including chuanxiong. Our findings indicated that $X Z T$ exerted its anticancer function by inhibiting the proliferation, invasion, and migration of glioma cells and inducing U251 glioma cell apoptosis in vitro. Similar results reported that the mean survival time and life span of mice suffering from experimental liver tumors were notably improved by $X Z T$ treatment compared to the control group. ${ }^{19}$ Therefore, $X Z T$ may have the potential to be a new therapeutic agent for patients with glioma.

$X Z T$ is a typical formulation for invigorating blood circulation and dissipating blood stasis and was first described by Yi Lin Gai Cuo, which was a monograph on TCM cases in Qing Dynasty in AD $1830 .{ }^{20}$ Previous studies have provided evidence that $X Z T$ is able to treat cardiovascular and liver diseases. For example, a study conducted by Zhou et a ${ }^{12}$ suggested that $X Z T$ could not only refrain collagen deposition but also exert its antiangiogenic effect on fibrotic liver through relieving hypoxia and preserving liver sinusoidal endothelial cell functions, thus restraining 
A

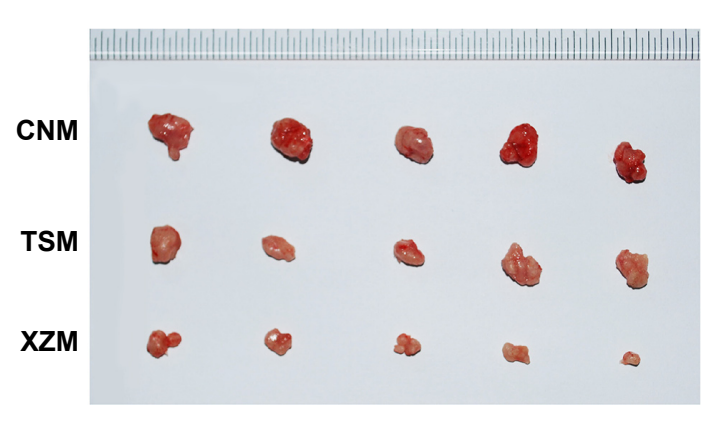

B

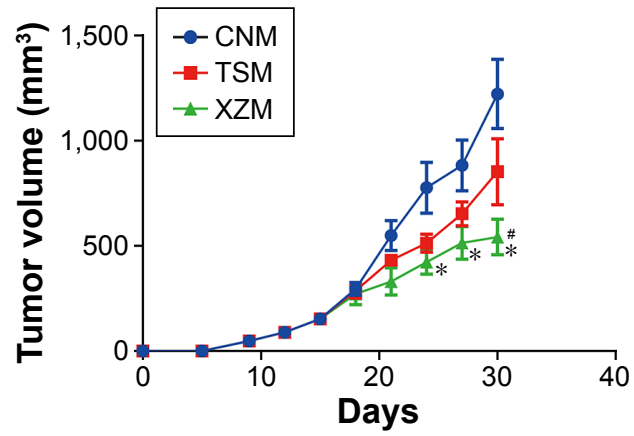

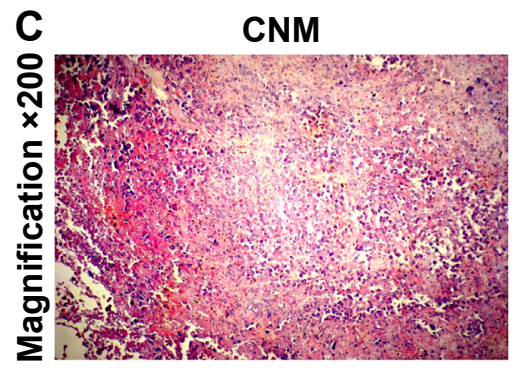

D
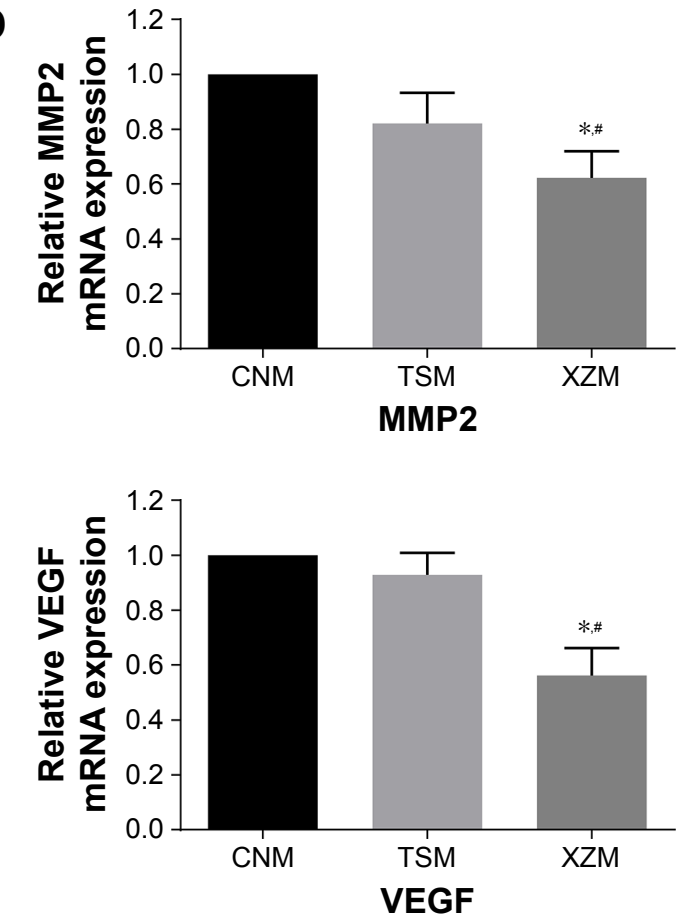

TSM

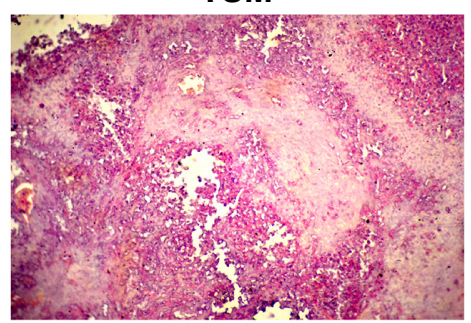

XZM

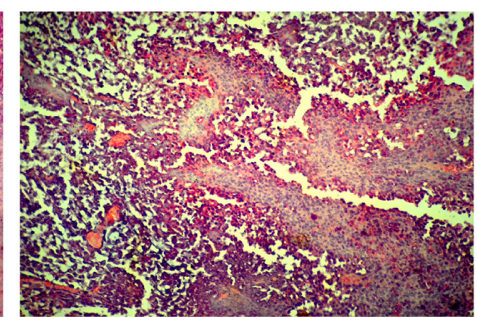

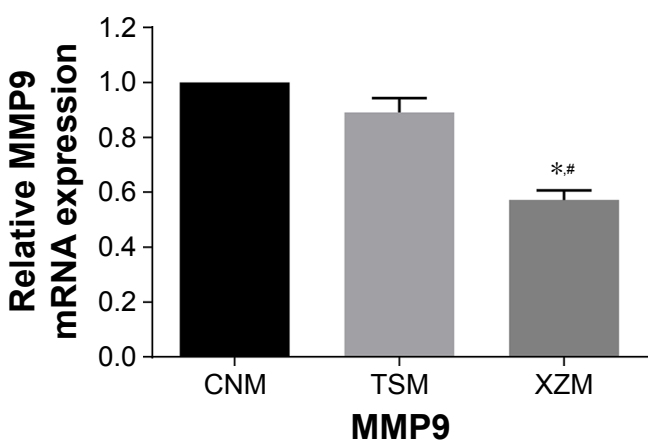

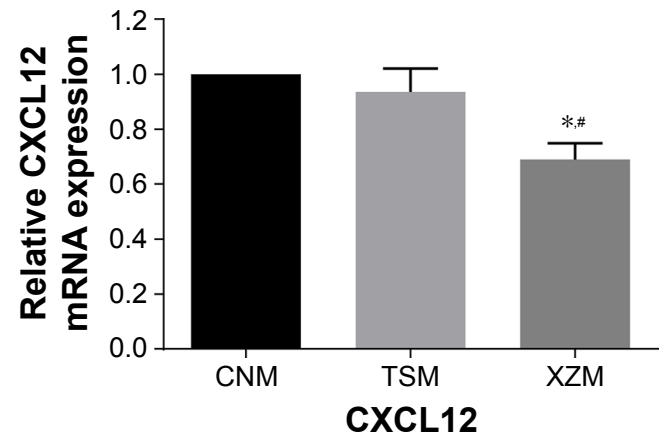

Figure 7 XZT inhibits the glioma tumor growth at 30 days after gavage in vivo.

Notes: (A) The representative photo of glioma tumors in each xenograft model group. (B) The tumor volumes decreased significantly with the group $X Z M$ compared to the group CNM or group TSM. *Versus group CNM, $P<0.05$; ${ }^{*}$ Group $X Z M$ versus group TSM, $P<0.05$. (C) The number was decreased significantly in the group $X Z M$ compared to the group CNM or group TSM analyzed by H\&E stain. Magnification $\times 200$. (D) Relative expressions of MMP2, MMP9, VEGF, and CXCLI2 were downregulated in xenograft mice of the group $X Z M$ compared to those of the group $C N M$ or group TSM. *Versus group $C N M, P<0.05$; ${ }^{*} G r o u p ~ X Z M$ versus group TSM, $P<0.05$. Group CNM, gavaging saline; group TSM, gavaging TST; group XZM, gavaging XZT.

Abbreviations: H\&E, hematoxylin and eosin; TST, TaohongSiwu Tang; XZT, XuefuZhuyu Tang.

liver fibrosis. Moreover, Hwang et $\mathrm{al}^{21}$ showed that $X Z T$ could significantly affect the progression of syndromes and inflammatory markers observed in patients with unstable angina pectoris after treating with percutaneous coronary intervention.
This study enabled us to discover that $X Z T$ remarkably upregulated the expression of TIMP1 and significantly downregulated expression of VEGF, VEGFR, CXCR4, CXCL12, MMP9, and MMP2 in U251 glioma cells in vitro, implying that $X Z T$ may affect glioma cells via regulating TME. 
Likewise, our investigations in vivo revealed that tumor volumes in group XZM were significantly lower than those in group CNM and group TSM. Moreover, the expressions of VEGF, CXCL12, MMP9, and MMP2 were downregulated in group XZM compared with group CNM and group TSM, which was consistent with the results obtained from RT-PCR and Western blotting in vitro. Our findings of CXCR4/CXCL12 pathway were agreed with those by Chen et al, which suggested that downregulated expression of chemokine receptor CXCR4 by TMP suppressed the activities of glioma cells, including proliferation, migration, and invasion, and reduced the release of glutamate from cerebral neurocytes. These results indicated that the CXCR4/CXCL12 pathway may be considered as a new and potential mechanism in which TMP-mediated tumor is depressed and neural functions are preserved. ${ }^{16}$

To date, growing studies have supported that TME is an emerging therapeutic target for glioma. For instance, Yang et $\mathrm{al}^{22}$ reported that the activation of CXCR4 could induce glioma growth in which c-AMP is accumulated in glioma cells, whereas the specific CXCR4 antagonist AMD 3465 is able to inhibit tumor growth both in vivo and in vitro, indicating that the CXCR4/CXCL12 axis may possess critical roles in tumor progression. Yang et al also demonstrated that high-LET carbon ion radiation was preferred to low-LET $\mathrm{X}$-rays with respect to suppressing the migration of glioma cells. Furthermore, their study showed that X-ray radiation enhanced the secretion of VEGF, MMP2, and MMP9, which can further degrade basement membranes and extracellular matrixes when tumor progression occurrs. ${ }^{23-26}$ Qazi et $\mathrm{al}^{27}$ also demonstrated that fluid shear stress may decrease the motility of glioma cells through modulating MMPs' activation and expression. Furthermore, Najbauer et al noted that VEGF could contribute to the generation of distended and glomerulus-like vessels in glioma cells after treating with MMPs. ${ }^{28}$ However, it is still difficult to explain whether inhibitory effects of XZT on VEGF/VEGFR, CXCR4/CXCL12, and TIMP1/MMP9/MMP2 pathway are independent or coadjusted. Therefore, further research is required to clarify the relationship between VEGF/VEGFR, CXCR4/CXCL12, and TIMP1/MMP9/MMP2 in the inhibitory mechanism of $X Z T$.

\section{Conclusion}

Our study suggested that XZT could possess many properties, including the inhibition of glioma cell proliferation, protection of neurons from excitotoxicity induced by glioma cells, and improving blood-brain barrier penetration. Apart from that, $X Z T$ may suppress the proliferation, invasion, migration, and growth of glioma cells through regulating the expression levels of microenvironment relative factors. Importantly, multilevels, multitargets, and multiple pathways were observed in the anticancer mechanism of XZT. These significant findings may provide a profound explanation for the molecular basis of $X Z T$ in treating glioma and may direct future research, which will be able to clarify the clinical benefits of $X Z T$.

\section{Acknowledgment}

This study was funded by the Natural Science Foundation of China (number 81072907) and the Science and Technology Program of Guangzhou, People's Republic of China (number 20150429090440904).

\section{Disclosure}

The authors report no conflicts of interest in this work.

\section{References}

1. Morgan LL. The epidemiology of glioma in adults: a "state of the science" review. Neuro Oncol. 2015;17(4):623-624.

2. Ohgaki H, Kleihues P. Epidemiology and etiology of gliomas. Acta Neuropathol. 2005;109(1):93-108.

3. Jovcevska I, Kocevar N, Komel R. Glioma and glioblastoma-how much do we (not) know? Mol Clin Oncol. 2013;1(6):935-941.

4. Whipple CA. Tumor talk: understanding the conversation between the tumor and its microenvironment. Cancer Cell Microenviron. 2015;2(2): e773.

5. Wurth R, Bajetto A, Harrison JK, Barbieri F, Florio T. CXCL12 modulation of CXCR4 and CXCR7 activity in human glioblastoma stem-like cells and regulation of the tumor microenvironment. Front Cell Neurosci. 2014;8:144.

6. Lu KV, Chang JP, Parachoniak CA, et al. VEGF inhibits tumor cell invasion and mesenchymal transition through a MET/VEGFR2 complex. Cancer Cell. 2012;22(1):21-35.

7. Hamerlik P, Lathia JD, Rasmussen R, et al. Autocrine VEGF-VEGFR2neuropilin-1 signaling promotes glioma stem-like cell viability and tumor growth. J Exp Med. 2012;209(3):507-520.

8. Remillard TC, Bratslavsky G, Jensen-Taubman S, Stetler-Stevenson WG, Bourboulia D. Molecular mechanisms of tissue inhibitor of metalloproteinase 2 in the tumor microenvironment. Mol Cell Ther. 2014; 2:17.

9. Yi GZ, Qiu YQ, Xiao Y, Yuan LX. The usefulness of xuefu zhuyu tang for patients with angina pectoris: a meta-analysis and systematic review. Evid Based Complement Alternat Med. 2014;2014: 521602.

10. Kubatka P, Kapinova A, Kruzliak P, et al. Antineoplastic effects of chlorella pyrenoidosa in the breast cancer model. Nutrition. 2015;31(4):560-569.

11. Kubatka P, Kapinova A, Kello M, et al. Fruit peel polyphenols demonstrate substantial anti-tumour effects in the model of breast cancer. Eur J Nutr. 2016;55(3):955-965.

12. Zhou YN, Sun MY, Mu YP, et al. Xuefuzhuyu decoction inhibition of angiogenesis attenuates liver fibrosis induced by $\mathrm{CCL}_{4}$ in mice. J Ethnopharmacol. 2014;153(3):659-666.

13. Blacher E, Ben Baruch B, Levy A, et al. Inhibition of glioma progression by a newly discovered CD38 inhibitor. Int J Cancer. 2015;136(6): 1422-1433.

14. Ling CQ, Yue $X Q$, Ling $C$. Three advantages of using traditional chinese medicine to prevent and treat tumor. J Integr Med. 2014;12(4):331-335.

15. Zhai XF, Chen Z, Li B, et al. Traditional herbal medicine in preventing recurrence after resection of small hepatocellular carcinoma: a multicenter randomized controlled trial. J Integr Med. 2013;11(2):90-100. 
16. Chen Z, Pan X, Georgakilas AG, et al. Tetramethylpyrazine (TMP) protects cerebral neurocytes and inhibits glioma by down regulating chemokine receptor CXCR4 expression. Cancer Lett. 2013;336(2): 281-289.

17. Fu YS, Lin YY, Chou SC, et al. Tetramethylpyrazine inhibits activities of glioma cells and glutamate neuro-excitotoxicity: potential therapeutic application for treatment of gliomas. Neuro Oncol. 2008; 10(2):139-152.

18. Yu K, Chen Z, Pan X, et al. Tetramethylpyrazine-mediated suppression of C6 gliomas involves inhibition of chemokine receptor CXCR4 expression. Oncol Rep. 2012;28(3):955-960.

19. You JS, Huang HF, Hau DM. Effects of xuefu zhuyu tang and mitomycin C on liver tumors in mice. Chang Gung Med J. 2003;26(6): $417-424$.

20. Lin F, Chen BL, Wang YZ, et al. In vitro angiogenesis effect of xuefu zhuyu decoction (血府逐疼汤) and vascular endothelial growth factor: a comparison study. Chin J Integr Med. Epub 2015 Aug 14.

21. Hwang BM, Noh EM, Kim JS, et al. Curcumin inhibits UVBinduced matrix metalloproteinase- $1 / 3$ expression by suppressing the MAPK-p38/JNK pathways in human dermal fibroblasts. Exp Dermatol. 2013;22(5):371-374.
22. Yang L, Jackson E, Woerner BM, Perry A, Piwnica-Worms D, Rubin JB. Blocking CXCR4-mediated cyclic amp suppression inhibits brain tumor growth in vivo. Cancer Res. 2007;67(2):651-658.

23. Kil WJ, Tofilon PJ, Camphausen K. Post-radiation increase in VEGF enhances glioma cell motility in vitro. Radiat Oncol. 2012;7:25.

24. Meng X, Liu Y, Liu B. Glutathione S-transferase M1 null genotype meta-analysis on gastric cancer risk. Diagn Pathol. 2014;9:122.

25. Parthymou A, Kardamakis D, Pavlopoulos I, Papadimitriou E. Irradiated C6 glioma cells induce angiogenesis in vivo and activate endothelial cells in vitro. Int J Cancer. 2004;110(6):807-814.

26. Wild-Bode C, Weller M, Rimner A, Dichgans J, Wick W. Sublethal irradiation promotes migration and invasiveness of glioma cells: implications for radiotherapy of human glioblastoma. Cancer Res. 2001;61(6):2744-2750.

27. Qazi H, Shi ZD, Tarbell JM. Fluid shear stress regulates the invasive potential of glioma cells via modulation of migratory activity and matrix metalloproteinase expression. PLoS One. 2011;6(5):e20348.

28. Najbauer J, Huszthy PC, Barish ME, et al. Cellular host responses to gliomas. PLoS One. 2012;7(4):e35150.
OncoTargets and Therapy

\section{Publish your work in this journal}

OncoTargets and Therapy is an international, peer-reviewed, open access journal focusing on the pathological basis of all cancers, potential targets for therapy and treatment protocols employed to improve the management of cancer patients. The journal also focuses on the impact of management programs and new therapeutic agents and protocols on

\section{Dovepress}

patient perspectives such as quality of life, adherence and satisfaction. The manuscript management system is completely online and includes a very quick and fair peer-review system, which is all easy to use. Visit http://www.dovepress.com/testimonials.php to read real quotes from published authors. 